Marquette University

e-Publications@Marquette

Physics Faculty Research and Publications

Physics, Department of

6-1-2010

Dynamic Notch Pinning Fields for Domain Walls in Ferromagnetic Nanowires

Andrew Kunz

Marquette University, andrew.kunz@marquette.edu

Jonathan D. Priem

Marquette University

Published version. IEEE Transactions on Magnetics, Vol. 46, No. 6 (June 2010), DOI. (C) 2010 Institute of Electrical and Electronics Engineers. Used with permission. 


\title{
Dynamic Notch Pinning Fields for Domain Walls in Ferromagnetic Nanowires
}

\author{
Andrew Kunz and Jonathan D. Priem \\ Physics Department, Marquette University, Milwaukee, WI 53233 USA
}

\begin{abstract}
Artificial defects such as notches and antinotches are often attached to magnetic nanowires to serve as trapping (pinning) sites for domain walls. The magnetic field necessary to release (depin) the trapped domain wall from the notch depends on the type, geometric shape, and dimensions of the defect but is typically quite large. Conversely we show here that for some notches and antinotches there exists a much smaller driving field for which a moving domain wall will travel past the defect without becoming trapped. This dynamic pinning field also depends on the type, geometric shape and defect dimensions. Micromagnetic simulation is used to investigate both the static and dynamic pinning fields and their relation to the topologic structure of the domain wall.
\end{abstract}

Index Terms-Magnetic devices, magnetic domains, magnetization reversal.

\section{INTRODUCTION}

$\mathbf{T}$ HE DYNAMIC properties of magnetic domain walls moving in ferromagnetic nanowires are being investigated due to their potential for applications in logic, sensing, and memory devices [1], [2]. In these devices it is important to know and control the location of the domain wall along the wire. There is a significant amount of research underway to describe the magnetic field necessary to remove domain walls from artificial defects such as notches cut into the wire, and antinotches attached to the wire as these defects can be used to hold domain walls at specific known locations [3]-[6]. The notches typically serve as strong pinning sites as large magnetic fields are necessary to release the domain wall from the notch. This depinning field is typically much larger than the Walker breakdown field which results in low average domain wall speeds [7]. It has been shown that current pulses of the correct length and collisions of domain walls can also be used to release the domain walls from the notches with much smaller fields [8], [9]. The usefulness of domain wall collisions for the depinning process in a device depends in part on the ability of a domain wall to move past a notch without being captured, otherwise a domain wall must exist at every notch. In this paper we report on a dynamic notch pinning potential which depends on the strength of the driving field, and therefore the speed of the domain wall as it approaches a defect. Fast moving domain walls are able to pass a notch that is able to capture and hold slower moving walls. The large depinning field ensures that the wall will remain in the notch when reasonable strength fields are applied leading to a new technique for writing information stored in a domain wall configuration.

\section{Simulation Details}

We simulate the motion and potential trapping of a domain wall with standard three-dimensional micromagnetic simula-

Manuscript received October 29, 2009; revised December 10, 2009; accepted January 12, 2010. Current version published May 19, 2010. Corresponding author: A. Kunz (e-mail: andrew.kunz@marquette.edu).

Digital Object Identifier 10.1109/TMAG.2010.2041044 tion. The simulations follow the standard Landau-Lifshitz equation of motion for the magnetic moments $m$ in the wire

$$
\frac{\partial \vec{m}}{d t}=-\gamma \vec{m} \times H-\frac{\alpha \gamma}{M_{s}} \vec{m} \times(\vec{m} \times \vec{H})
$$

where $\gamma$ is the gyromagnetic ratio, $M_{s}$ is the saturation magnetization and $H$ is the total magnetic field acting on a magnetic moment [10]. The materials parameters are for permalloy. We simulate wires with a minimum length of 3 microns and a $100 \times 4 \mathrm{~nm}^{2}$ rectangular cross-section. The wire is discretized into identical cubes and integrated with a 4 th order predictor corrector technique with a simulated integration time step of less than a picosecond and a damping parameter of $\alpha=0.008$.

To put the walls in motion, magnetic fields are applied to free domain walls along the long axis of the wire. We keep the moving wall field strength below the so-called Walker breakdown field to maintain the domain walls of a known shape and because this is the field range for which the walls move the quickest. We place notches and antinotches along the wire to serve as potential trapping sites for the domain wall.

\section{RESUlTS AND DISCUSSION}

In narrow, thin ferromagnetic nanowires the magnetic moments lie in the plane of the film and are oriented along the long axis direction. A transverse domain wall separates oppositely oriented head-to-head, or tail-to-tail domain walls [11]. In Fig. 1 we show the final state for two triangular notch/anti-notch configurations each with two different domain wall magnetizations (up or down in the figure), separating tail-to-tail domains. In each case a domain wall entered from the left side of the figure and was driven to the right by a 15 Oe field placed to the left and parallel to the long axis of the wire. The final location of the domain wall depends on the domain wall orientation, notch location and notch type. The pinning potential of the defect can be summarized best with a simple topologic model [12]. We have labeled the sign of the topologic half charge on each end of the domain wall (a complete domain wall has a net topologic charge of 0 ). We see that positive charges are strongly pinned at antinotches, whereas negative charges are pinned strongly at notches.

If the wall reaches the right most defect it must pass the other potential trapping sites. The dynamic wall behavior in the region 


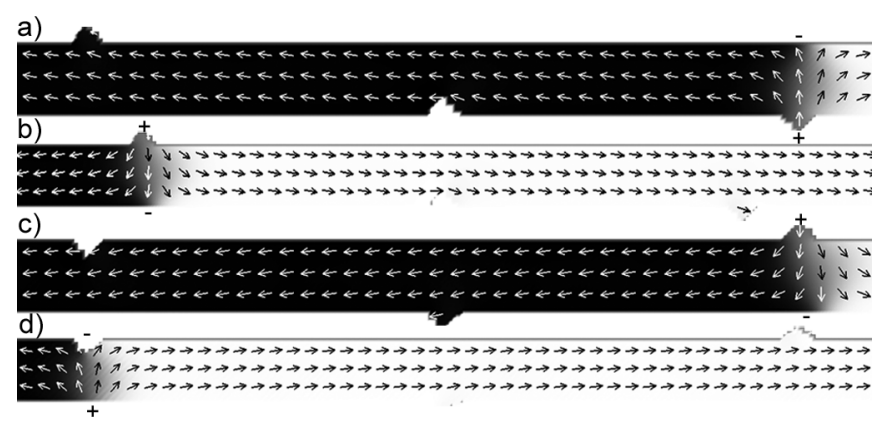

Fig. 1. Images a) and b) show the final simulated magnetic structure for a tail-to-tail domain wall driven from left to right in the figure for a given antinotch and notch configuration. The topologic charge structure of the domain wall is placed on the figure. Images c) and d) show the same for a different notch configuration. Notches trap negative topologic charges and antinotches trap positive charge.

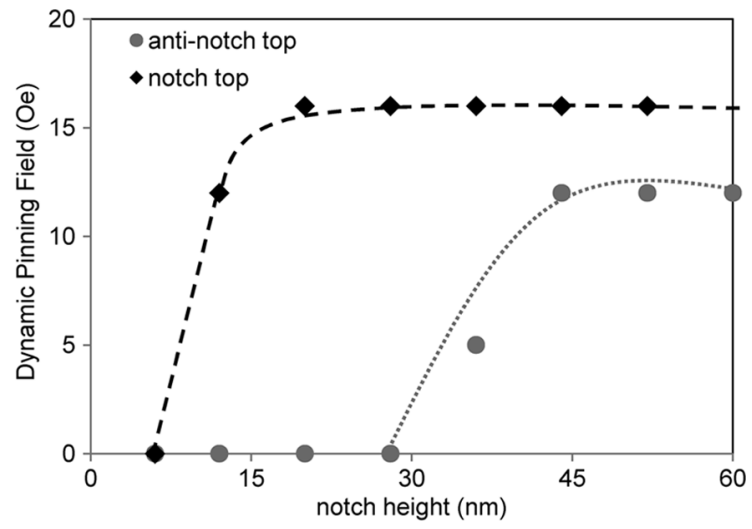

Fig. 2. Dynamic pinning fields as a function of notch depth (or antinotch height) for a negative topologic charge. For small defect sizes the domain walls can be driven past notches without being trapped, a $12 \mathrm{~nm}$ notch depth and 36 $\mathrm{nm}$ antinotch heights are required to trap a moving domain wall. The dashed lines are guides for the eye to help follow the trends.

of a potential pinning site really depends on whether or not the wall will be captured. When the wall passes a site, it does so with its magnetic structure almost unaffected with at most a small oscillation. However when a wall is captured the free end of the wall continues along, showing domain wall growth before the wall is snapped back toward the pinning site.

We explored the ability of a moving domain wall to move past a defect as a function of the type of notch and the dimensions of the notch for the given domain wall configuration. In Fig. 2 we summarize the driving field necessary to move a domain wall past a notch or antinotch, placed on the top edge of the nanowire, as a function of the depth of the notch. A head to head domain wall with magnetic moments pointing down is driven from left to right along the wire. The orientation of magnetic moments places the negative topologic charge on the top of the wire and the positive topologic charge on the bottom of the wire such as that shown in Fig. 1(b) and (c). The notch is a more effective pinning site in this configuration but the antinotch is still a weak trapping site. In short there is a different strength of interaction for notches and antinotches with positive and negative defects. In Fig. 3 we show the results of our simulations which give the field necessary to remove the now pinned wall from the notch or antinotch. The different field values between Fig. 2 and Fig. 3

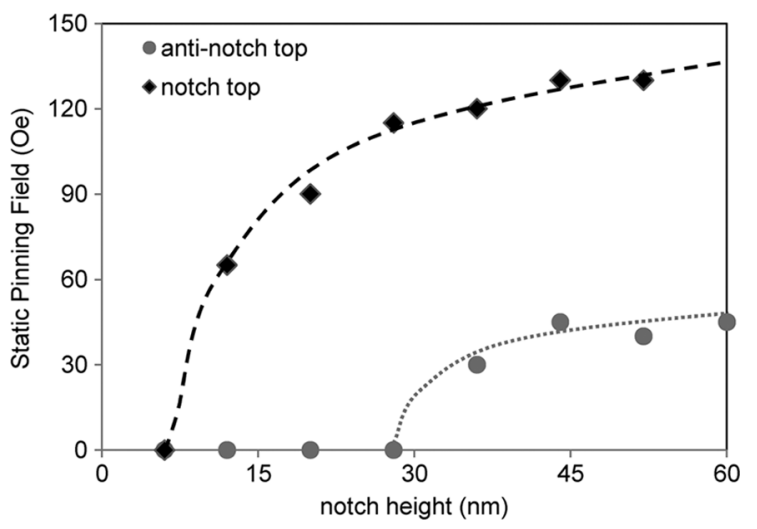

Fig. 3. Static pinning fields as a function of notch depth (or antinotch height) for a negative topologic charge. For domain walls trapped in notches (see Fig. 2) large fields are necessary to remove the walls. The lines are guides for the eye to help follow the trends.

demonstrates that there is a significant difference in the static pinning field which holds the wall in place and the dynamic pinning field which we define as the minimum field necessary to move a domain wall past a defect. The overall relationship remains the same in that the notch is a strong trap for a negative defect both in catching and releasing a wall. This behavior of Figs. 2, 3 is confirmed by placing the notch and antinotch on the bottom of the wire. The results are essentially reversed with the antinotch trapping the positive wall charge more effectively.

The difference in the interaction strength means that for shallow defects there is a field at which the domain walls can be driven fast enough to blow past a notch without becoming trapped. In Fig. 2 we show that a $12 \mathrm{~nm}$ notch depth (and a $36 \mathrm{~nm}$ antinotch height) is necessary to begin to trap the wall although the speed at which the wall approaches the notch allows the trapping to occur or not occur. We have confirmed this behavior by applying transverse in-plane fields to the domain wall driving field. A transverse field can be used to significantly speed up, or slow down, domain walls driven by identical driving fields [13]. In this case the driving field was used to increase the speed of a domain wall which allowed it to pass a notch where it was trapped without the driving field. Energetically this means the moving domain wall carries sufficient kinetic energy to climb the potential barrier for full magnetization reversal that the defect creates. As the depth of the defects increase, a domain wall is no longer able to pass the notch. The maximum driving field of 16 Oe corresponds to the Walker field above which the transverse domain walls undergo complicating transformations.

We see a similar, although reversed behavior when the depth of the notch is kept constant and the width of the notch is varied. In this case as the width increases, the pinning behavior decreases. This is expected because as the width increases the wire edge becomes effectively smooth.

The ability of a moving domain wall to either be captured or pass a notch in a wire allows for a simple technique to write domain walls in a known configuration. In Fig. 4 we show a few simulated images (Fig. 4(a)-(c)) which lead to the final magnetic states for a series of domain walls (Fig. 4(d)-(f)) injected at low fields from the pad on the left end of the wire [14]. There are 


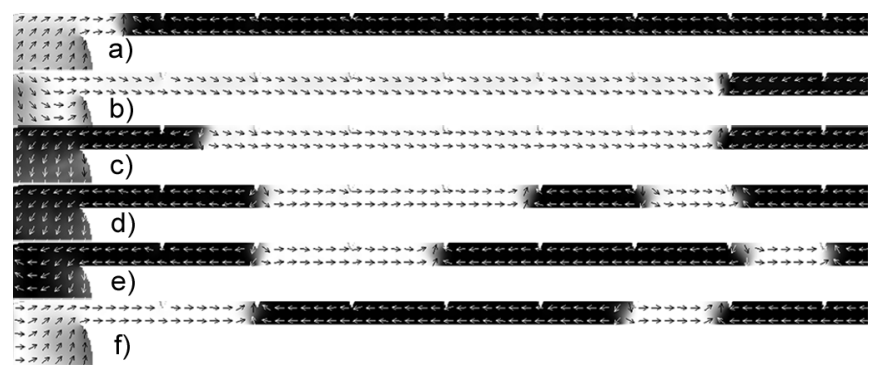

Fig. 4. Static and dynamic pinning allows for precise control of a domain wall. Walls can be injected (a), driven past notches (b) and placed where you want them as other walls are injected into the wire (c). Figs. (d)-(f) show three different final domain wall configurations which could be considered bit sequences.

eight notches placed on the upper edge of the wire; the domain walls are written from right to left. As shown in Fig. 4(a) the right most domain wall passed by each of the first six notches before being captured at the seventh notch. An additional in-plane transverse magnetic field was used to keep the domain wall speed high until it passed the sixth notch [13]. Once the notch passed the sixth notch the transverse field was turned off but the driving field was left on to drive the wall into the final notch as shown in Fig. 4(b). The next wall was injected, Fig. 4(c), and driven into its final location with a combined driving and transverse field until it passed the notch before the pinning site, without affecting the position of the first wall. Each domain wall was written in the same way leaving the final configuration as shown in Fig. 4(d). Fig. 4(e), (f) are two different final binary bit sequences. Each configuration was written in a simulation time of less than $25 \mathrm{~ns}$.

In conclusion there are two important fields to know when moving and capturing domain walls with artificial defects. A dynamic pinning field determines the minimum field necessary to pass a moving domain wall past a defect without being captured and the static pinning field is the minimum field needed to remove a captured domain wall from the defect. The specific value of the field depends on the topologic structure of the domain wall and the dimensions and type of defect but the static pinning field is always much stronger than the dynamic pinning field. By varying the wall speed domain walls can be moved with magnetic fields in one direction and placed reliably at specific locations in a wire.

\section{ACKNOWLEDGMENT}

This work was supported by the National Science Foundation under Grant DMR-0706194.

\section{REFERENCES}

[1] S. S. P. Parkin, M. Hayashi, and L. Thomas, "Magnetic domain-wall racetrack memory," Science, vol. 320, pp. 190-194, 2008.

[2] D. A. Allwood, "Magnetic domain-wall logic," Science, vol. 309, pp. 1688-1692, 2005.

[3] S.-H. Huang and C.-H. Lai, "Domain wall depinning by controlling its configuration at notch," Appl. Phys. Lett., vol. 95, p. 032505, 2009.

[4] L. K. Bogart, "Dependence of domain wall pinning potential landscapes on domain wall chirality and pinning site geometry in planar nanowires," Phys. Rev. B, vol. 79, p. 054414, 2009.

[5] D. Petit, "Mechanism for domain wall pinning and potential landscape modification by artificially patterned traps in ferromagnetic nanowires," Phys. Rev. B, vol. 79, p. 214405, 2009.

[6] D. Petit, "Domain wall pinning and potential landscapes created by constrictions and protrusions in ferromagnetic nanowires," $J$. Appl. Phys., vol. 103, p. 114307, 2008

[7] N. L. Schryer and L. R. Walker, "The motion of $180^{\circ}$ domain walls in uniform dc magnetic fields," J. Appl. Phys., vol. 45, pp. 5406-5421, 1974.

[8] M. Hayashi, "Dependence of current and field driven depinning of domain walls on their structure and chirality in permalloy nanowires," Phys. Rev. Lett., vol. 97, p. 207205, 2006

[9] A. Kunz, "Field Induced domain wall collisions in thin magnetic nanowires," Appl. Phys. Lett., vol. 94, p. 132502, 2009.

[10] Micromagnetic Simulator ver. 2.61 [Online]. Available: http://llgmicro.home.mindspring.com

[11] R. D. McMichael and M. J. Donahue, "Head to head domain wall structure in thin magnetic strips," IEEE Trans. Magn., vol. 33, no. 5, pp. 4167-4169, Oct. 1997.

[12] O. Tchernyshyov and G.-W. Chern, "Fractional vortices and composite domain walls in flat nanomagnets," Phys. Rev. Lett., vol. 95, p. 197404 2005.

[13] A. Kunz and S. C. Reiff, "Enhancing domain wall speeds in nanowires with transverse magnetic fields," J. Appl. Phys., vol. 103, p. 07D903, 2008.

[14] A. Kunz and S. C. Reiff, "Dependence of domain wall structure for low field injection into magnetic nanowires," Appl. Phys. Lett., vol. 94, p. 192504, 2009. 